Radiation effects and defects in lithium borate crystals

This article has been downloaded from IOPscience. Please scroll down to see the full text article.

2010 IOP Conf. Ser.: Mater. Sci. Eng. 15012016

(http://iopscience.iop.org/1757-899X/15/1/012016)

View the table of contents for this issue, or go to the journal homepage for more

Download details:

IP Address: 93.88.179.11

The article was downloaded on 02/12/2010 at 12:46

Please note that terms and conditions apply. 


\title{
Radiation effects and defects in lithium borate crystals
}

\author{
Igor N Ogorodnikov, Nikita E Poryvay and Vladimir A Pustovarov \\ Ural Federal University, Mira Street, 19, Ekaterinburg 620002, Russia \\ E-mail: igor.ogorodnikov@bk.ru
}

\begin{abstract}
The paper presents the results of a study of the formation and decay of lattice defects in wide band-gap optical crystals of $\mathrm{LiB}_{3} \mathrm{O}_{5}(\mathrm{LBO}), \mathrm{Li}_{2} \mathrm{~B}_{4} \mathrm{O}_{7}$ (LTB) and $\mathrm{Li}_{6} \mathrm{Gd}\left(\mathrm{BO}_{3}\right)_{3}$ (LGBO) with a sublattice of mobile lithium cations. By means of thermoluminescence techniques, and luminescent and absorption optical spectroscopy with a nanosecond time resolution under excitation with an electron beam, it was revealed that the optical absorption in these crystals in the visible and ultraviolet spectral ranges is produced by optical hole-transitions from the local defect level to the valence band states. The valence band density of the states determines mainly the optical absorption spectral profile, and the relaxation kinetics is rated by the interdefect non-radiative tunnel recombination between the trapped-hole center and the $\mathrm{Li}^{0}$ trapped-electron centers. At $290 \mathrm{~K}$, the $\mathrm{Li}^{0}$ centers are subject to thermally stimulated migration. Based on experimental results, the overall picture of thermally stimulated recombination processes with the participation of shallow traps was established for these crystals.
\end{abstract}

\section{Introduction}

The lithium borate crystals $\mathrm{LiB}_{3} \mathrm{O}_{5}$ (LBO), $\mathrm{Li}_{2} \mathrm{~B}_{4} \mathrm{O}_{7}$ (LTB), $\mathrm{Li}_{6} \mathrm{Gd}\left(\mathrm{BO}_{3}\right)_{3}$ (LGBO) are typical representatives of an extensive class of wide band-gap dielectrics with mobile cations. Recently these optical materials have been intensively investigated both from the point of view of their fundamental properties, and with respect of their practical applications in the fields of radiation detectors and transformers and optical wave-guides operating in a broad spectral range from the visible area to the vacuum ultraviolet region. All the crystals are highly transparent down to the cutoff wavelength near $160 \mathrm{~nm}$ and their band gaps were estimated as $8.8-9 \mathrm{eV}[1,2]$. The lithium borate crystals have recently become promising candidates for the realization of neutron imaging using scintillation methods. Despite variations in chemical composition, these materials have many similar properties. In particular, they have a reduced symmetry of the crystal lattice and a complex elementary cell. The crystal structure of each material consists of the appropriate boron-oxygen anionic groups and lithium cations. The lithium cations occupy the voids in the continuous boron-oxygen network. A distinctive feature of these materials is the sharp contrast between the strong covalent chemical bonds inside the anionic groups and the comparatively weak ionic bonds between the lithium cations and appropriate anionic groups.

The presence in the lattice of weakly bonded lithium cations in combination with a robust anionic frame should dramatically affect dynamics of electronic excitations and creation of radiation induced lattice defects. This problem gets a special sharpness in the case of small radius lithium cations. However, despite the obvious importance of the considered phenomenon, a systematic study of formation and evolution of the lattice defects in the lithium sublattice of these crystals was not carried out prior to the beginning of our work.

There are only few works devoted to the study of defects and thermally stimulated recombination processes in these crystals below room temperatures. Namely, the luminescence and optical spectroscopy 
of defects [3-5], electron spin resonance (ESR) study of low-temperature lattice defects in LBO [6,7], thermally stimulated luminescence in LBO [8] have been investigated. The main goal of the present work is a study of the radiation effects and defects in the lithium borate crystals over the temperature range from 90 to $500 \mathrm{~K}$.

\section{Experimental details}

The samples of lithium borate crystals were plane-parallel transparent plates measuring $6 \times 5 \times 1 \mathrm{~mm}^{3}$. The LBO and LTB crystals were grown at the Institute of Geology and Mineralogy, Siberian branch of Russian Academy of Sciences (Novosibirsk). The LGBO crystals were grown at the Institute of Single Crystals, National Academy of Sciences of Ukraine (Kharkov), by the Czochralski method in air atmosphere [9].

For the measurements of the thermally stimulated luminescence (TL) the samples were placed in a vacuum cryostat. The temperature of the sample could be linearly changed between 90 and $500 \mathrm{~K}$ by means of a special temperature controller. TL glow-curves were measured with a heating rate of $0.3 \mathrm{~K} \cdot \mathrm{s}^{-1}$ after irradiation of the samples at $90 \mathrm{~K}$ with an electron beam $\left(E_{\mathrm{e}}=200 \mathrm{keV}\right.$, fluence $\Phi=$ $10^{14} \mathrm{~cm}^{-2}$ ). The FEU-106 type photomultiplier tube was used to record TL-intensity.

The experimental setup and the main characteristics of the luminescence and absorption spectroscopy with a nanosecond-scale time-resolution employed are described in considerable detail in [10]. The pulsed excitation of the samples was done by the means of a pulsed electron beam with an electron energy of $200 \mathrm{keV}$, an excitation pulse duration of $15 \mathrm{~ns}$, and an excitation pulse power of $4-40 \mathrm{MW} \cdot \mathrm{cm}^{-2}$.

\section{Experimental results}

TL glow curves of the lithium borate crystals have been recorded after irradiation at $90 \mathrm{~K}$ with the same dose (figure 1). The observed TL glow curves are well fitted by the general-order kinetic equation [11] using ORIGIN program. The trapping parameters of principal TL-peaks for all the crystals have been obtained and were summarized in table 1. All three glow curves comprise the main intense complex TL peak at 100-130 K, which can be well fitted with either two (LBO, LGBO) or three peaks (LTB) (table 1). In the temperature range from 200 to $320 \mathrm{~K}$ the observed TL glow-curves are quite different (figure 1).

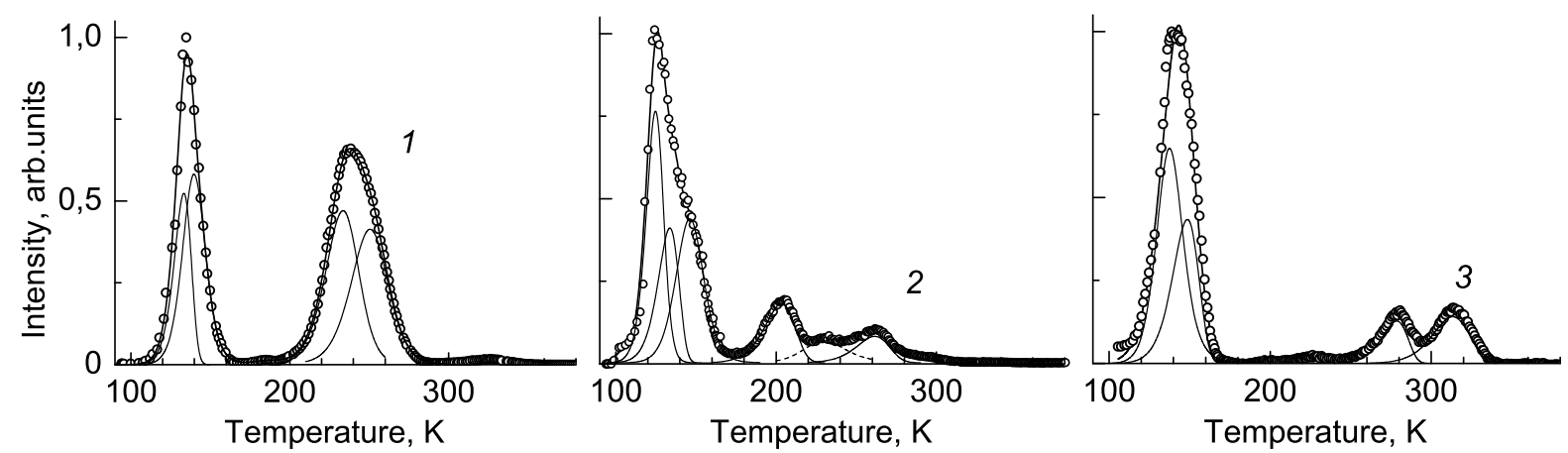

Figure 1. TL glow curves for crystals of LBO - (1), LTB - (2), and LGBO - (3) measured with a linear heating rate $0.3 \mathrm{~K} \cdot \mathrm{s}^{-1}$ after irradiation at $90 \mathrm{~K}$

The LBO crystal manifests an intensive complex TL peak at about $240 \mathrm{~K}$, which can be decomposed into two TL peaks, and two low-intensity TL peaks at 180 and $330 \mathrm{~K}$. In contrast to this, the LTB and LGBO crystals manifest only several low-intensity TL peaks in this temperature range (table 1).

Excitation of the lithium borate crystals with a single nanosecond-range electron pulse brought about a short drop in optical transparency of the crystal, i.e., an induced transient optical absorption event (TOA). The decay time of this absorption exceeds by many orders of magnitude the length of the exciting 
Table 1. Trapping parameters of principal TL-peaks in lithium borate crystals

\begin{tabular}{lccccc}
\hline \multirow{2}{*}{ Crystal } & $T_{\mathrm{m}}, \mathrm{K}$ & $E, \mathrm{eV}$ & $S, \mathrm{~s}^{-1}$ & $p$ & $I_{\mathrm{m}}$, arb. units \\
\hline \multirow{4}{*}{ LBO } & 133 & 0.29 & $5 \cdot 10^{9}$ & 1.0 & 52 \\
& 140 & 0.30 & $5 \cdot 10^{9}$ & 1.7 & 58 \\
& 234 & 0.54 & $2 \cdot 10^{10}$ & 1.5 & 47 \\
& 250 & 0.57 & $1 \cdot 10^{10}$ & 1.5 & 41 \\
\hline \multirow{4}{*}{ LTB } & 124 & 0.29 & $4 \cdot 10^{10}$ & 1.4 & 77 \\
& 134 & 0.23 & $3 \cdot 10^{7}$ & 1.0 & 41 \\
& 147 & 0.27 & $8 \cdot 10^{7}$ & 1.6 & 44 \\
& 204 & 0.38 & $9 \cdot 10^{7}$ & 1.0 & 18 \\
\multirow{4}{*}{ LGBO } & 228 & 0.48 & $1 \cdot 10^{9}$ & 2.0 & 7 \\
& 262 & 0.60 & $1 \cdot 10^{10}$ & 1.1 & 9 \\
\hline
\end{tabular}

Note. $T_{\mathrm{m}}$ - temperature position of the TL peak; $I_{\mathrm{m}}$ - intensity of TL-peak in percents of the highest TL intensity in the temperature range studied; $E$-activation energy; $S$ - pre-exponential factor; $p$ - kinetics order.

electron beam pulse. The broad (not unfolded) TOA spectrum (figure 2) is located in the region of optical transparency of the crystal.

Figure 2 plots the TOA decay kinetics. An analysis of the kinetics revealed that while at $T=290 \mathrm{~K}$ slow monotonic relaxation of induced optical density occurs within a broad ( 9 decades) time interval, the dynamic range of its variation is relatively small.

One could isolate two decay-time regions differing in the decay pattern. In the micro- and millisecond region, experimental curves allow readily rectification within 3-4 decay time decades when plotted in $\log -\log$ coordinates (figure 2). They can formally be described by the relation

$$
-\log D(t)=A+p \cdot \log t
$$

The exponent $p$ depends on decay time and lies within 0.04-0.20. Such properties are characteristic of interdefect tunneling recombination kinetics. In the case where the concentration of one of the recombination partners $N$ exceeds by far that of defects of another species $n$ which account for the observed TOA, one can write the equation of [12]

$$
n(t)=n_{0} \exp \left(-\frac{4}{3} \pi a^{3} N \ln ^{3}\left(v_{0} t\right)\right)
$$

where $v_{0}$ is the prefactor, and $a$ is one half of the Bohr radius, the parameters which determine the electron tunneling transfer probability $W$. For long decay times, the TOA kinetics of all the crystals also follows the straight line (1) but with an exponent $p=1$, which assumes a first-order hyperbolic relation

$$
D(t)=\frac{D_{\mathrm{h}}}{1+t / t_{\mathrm{h}}}
$$



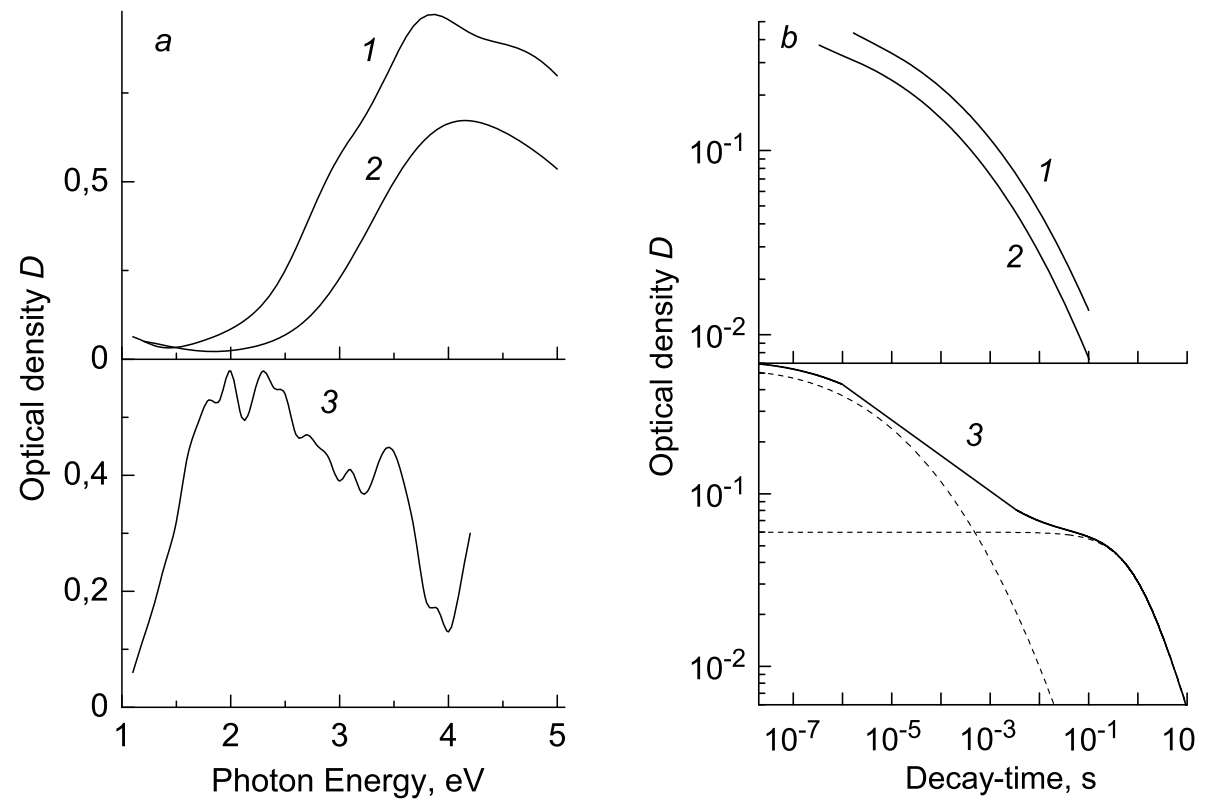

Figure 2. Transient optical absorption spectra $(a)$ and decay kinetics $(b)$ for crystals of LTB - (1), LBO - (2), and LGBO - (3), measured at $T=290 \mathrm{~K}$. Dashed lines plot the main constituents, and the solid lines are the experimental results

where $D_{\mathrm{h}}$ is the optical density corresponding to the initial concentration of the color centers accounting for the given TOA decay kinetics component, and $t_{\mathrm{h}}$ is the characteristic half-life time of these defects. The hyperbolic component is seen most clearly in the LGBO crystal for $t>10 \mathrm{~ms}$ (figure 2). However, numerical treatment of the TOA decay kinetics in LBO and LTB also reveals the presence of the lowintensity hyperbolic component. Figure 2 plots the results of approximation of the TOA decay kinetics with a sum of relations (2) and (3) for decay times extending over about nine decades.

An increase of excitation pulse power gives rise to an increase of the initial concentration of the radiation defects created by this pulse. This brings about a certain "shortening" of the TOA decay kinetics. When plotted in $\log -\log$ coordinates, the initial optical density $D(0)$, which is proportional to the initial concentration of radiation defects, grows by a linear law with a slope close to unity throughout the spectral range covered (figure 3). This may suggest the non-impurity nature of the color centers and a common mechanism of breakup of the radiation defects accounting for the different TOA bands.

Heating of the crystal likewise brings about "shortening" of the TOA decay kinetics, but the initial optical density $D(0)$ does not exhibit here any noticeable changes. Both TOA decay kinetics components in the 1.0-4.0 eV region exhibit the same behavior with temperature. The temperature dependence of the tunnel component (2) is dominated by the prefactor $v_{0}(T)$. According to [13],

$$
v_{0}(T)=\frac{C}{T^{1 / 2}} \exp \left(-\frac{E_{\mathrm{a}}}{k_{\mathrm{b}} T}\right)
$$

where $C$ is a constant, $k_{\mathrm{b}}$ is the Boltzmann constant, and $E_{\mathrm{a}}$ is the tunneling activation energy. Fitting Eq. (2) for a fixed value $a^{3} N=4 \cdot 3 \cdot 10^{-4}$ yielded values of $v_{0}$ for various temperatures. Plotted in the Arrhenius coordinates, the relation $v_{0}(T) \cdot T^{1 / 2}$ can be rectified with the activation energies $E_{\mathrm{a}}$ (figure 3 ).

Figure 3 shows also the temperature dependence of the time constant $t_{\mathrm{h}}$ for the hyperbolic component of the LGBO TOA decay kinetics. This component obeys the Arrhenius law (5) with a thermal activation 

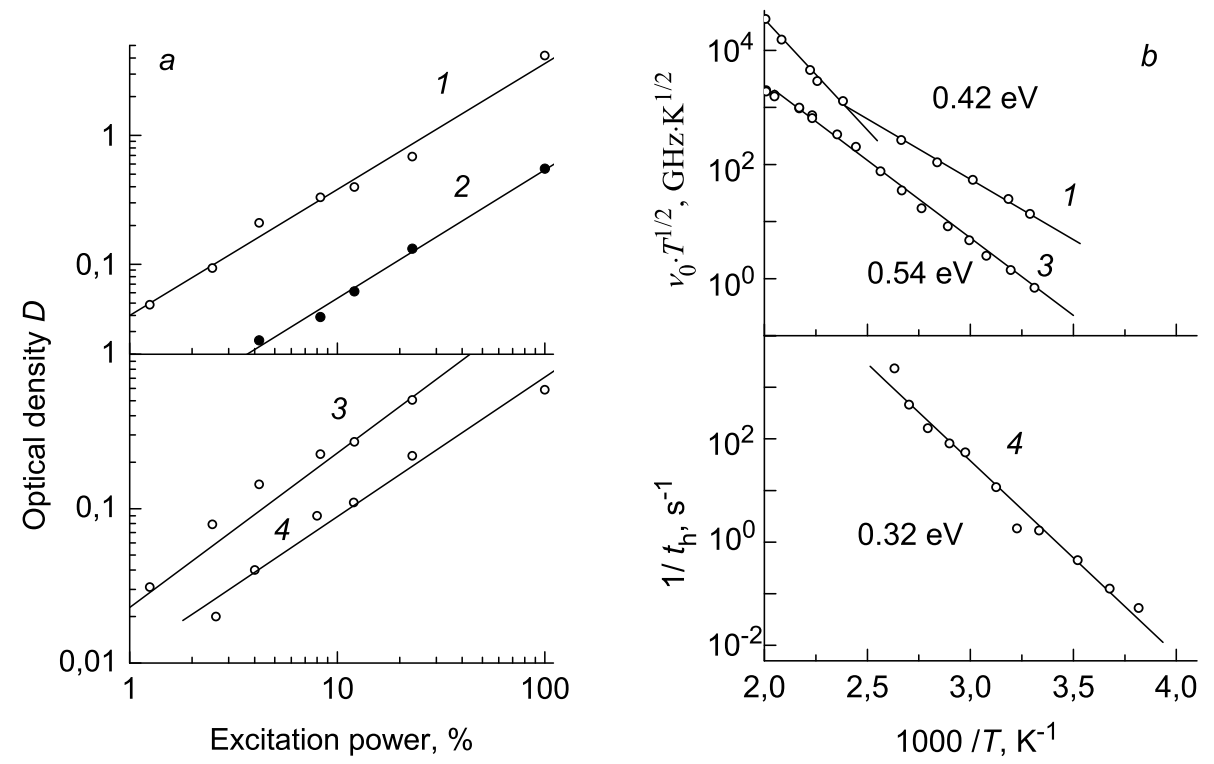

Figure 3. Optical density measured at $E_{\mathrm{m}}=3.8-(1,3,4), 2.1 \mathrm{eV}-(2)$, and $T=290 \mathrm{~K}$ immediately after termination of the excitation pulse and plotted vs. electron beam power (percents of the maximum power) $-(a)$ and a temperature dependence of parameters of the TOA decay kinetics $-(b)$ for crystals of LTB - (1,2), LBO - (3) and LGBO - (4)

energy $E_{\mathrm{a}}=0.32 \mathrm{eV}$ and a prefactor $\omega_{0}=4 \cdot 10^{5} \mathrm{~s}^{1}$.

$$
\frac{1}{t_{\mathrm{h}}(T)}=\omega_{0} \cdot \exp \left(-\frac{E_{\mathrm{a}}}{k_{\mathrm{b}} T}\right)
$$

\section{Discussion}

The common feature of the lithium borate crystals is a presence of the weakly bonded lithium cations. A comparatively weak radiation action at room temperature can create the radiation-induced Frenkel's pairs of the 'vacancy-interstitial atom' defects in the lithium cation sublattice of these crystals. A capture of the charge carriers on these defects leads to a creation of both the deep trapped-electron center $\mathrm{Li}^{0}$ and trapped-hole center $\mathrm{O}^{-}$.

The last center is defined as a small-radius hole polaron near the cation vacancy. Its origin was thoroughly studied for the LBO crystals by the electronic spin resonance technique: the angular dependencies of the ESR spectra [7,8], the non-isothermal relaxation of the ESR spectra [6]. All results bear witness to the fact that these defects manifest themselves in thermoluminescence: the complex TL peak at $100-130 \mathrm{~K}$ results from delocalization of holes from two competing traps originated initially from the $\mathrm{O}^{-}$-center. The trapped-hole centers being responsible for the complex TL peak at $240 \mathrm{~K}$ have similar origin. All these TL peaks in LBO are caused by delocalization of holes trapped on the $\mathrm{O}^{-}$centers. In all cases, these trapped-hole centers represent a hole localized on a $2 p$ oxygen orbital in the vicinity of the cation vacancy. Distinctions between these centers are determined by variations in their nearest surrounding. In our opinion, these types of $\mathrm{O}^{-}$-centers are typical for all lithium borate crystals, and observable experimentally differences in various recombination processes can be explained by only small distinctions in the parameters of these centers. The low-intensive TL peak at 180-200 K in lithium borates was earlier associated with the paramagnetic trapped-electron center $\mathrm{B}^{2+}[6]$.

Another reason for the existence of $\mathrm{O}^{-}$-center type diversity results from the presence of various impurities. Because of the charge compensation condition, the introduced heterovalent impurities can also stimulate the creation of lithium vacancies. These additional vacancies play the role of precursors 
for the creation of the shallow traps at a later stage. In doing so, the presence of the impurity-based defects can change the kinetics of recombination processes, shifts slightly the temperature positions of the TL peaks in the temperature range 90-300 K, and redistributes the TL intensity between these peaks in the lithium borate crystals. The origin of the observed TOA in the lithium borate crystals should be attributed to the formation of short-living radiation defects induced by the electron beam.

It should be noted that oxygen and boron vacancies and interstitial oxygen and boron ions in lithium borates can be created only under irradiation with particles of higher quantum energies than used in the present work. For example, the stable optical absorption spectra of LTB after irradiation with either neutrons or high-energy electrons have been revealed in [14,15]. From Ref. [15] it follows that $E_{\mathrm{e}}>1 \mathrm{Mev}$ is required for creation of the defects in the boron-oxygen sublattice of these crystals under irradiation with an electron beam.

An analysis of the crystallographic structure of borates suggests that the most probable effect of the action of the $200 \mathrm{keV}$ electron beam is only creation of Frenkel's pairs in the sublattice of weakly bound lithium cations, namely, of an interstitial lithium ion and a cation lithium vacancy. Subsequent trapping of the band electron by the lithium ion may result in formation of the $\mathrm{Li}^{0}$ electronic center, while trapping a band hole into $2 p$-orbitals of one of the oxygen ions surrounding a lithium vacancy gives rise to formation of an $\mathrm{O}^{-}$type hole center. Such mechanism of radiation defect formation was observed by us earlier to realize in LTB [3], LBO [4] and LGBO [5] lithium borate crystals.

It is obvious that the $\mathrm{O}^{-}$trapped-hole color centers responsible for the TOA are intrinsic lattice defects in borates, whose concentration plotted in log-log coordinates grows linearly with increasing excitation pulse power by nearly two orders of magnitude. This fact is beyond question. However, the mechanism of creation of these radiation-induced defects is not entirely clear. In fact, the $200 \mathrm{keV}$ electron beam can create the radiation-induced Frenkel's pairs of the 'vacancy-interstitial atom' defects in the lithium cation sublattice of these crystals. It is fairly easy to estimate that the concentration of the defects created through the impact mechanism under action of the electron beam pulse of $40 \mathrm{MW} \cdot \mathrm{cm}^{-2}$ should be approximately $10^{12} \mathrm{~cm}^{-2}$. At the same time, from the transient optical absorption spectra it follows that the concentration of the color centers may be as high as $10^{18} \mathrm{~cm}^{-3}[3,4]$. This means that the Frenkel defects in borates are created not only because of the knock-out (impact) mechanism but also because of the nonimpact mechanism. In our opinion, one possibility is that the mobile lithium cation has become slightly displaced from its regular lattice position under action of radiation. The off-center lithium ion can form a mobile dipole with the on site vacancy. A capture of an electron on this defect leads to the creation of a neutral $\mathrm{Li}^{0}$ atom, which can easily move away from the regular lattice position. This process can favor the creation of an elevated amount of the cation vacancies under action of an electron beam.

The broad optical absorption bands of hole centers in oxides are usually assigned to optical transitions between valence band states of the crystal and a local level of the hole center. It is known that the $\mathrm{O} 2 p$ - and B $2 p$-like hybridized states provide a major contribution to valence-band top states of the borates, while levels of the conduction band bottom derive from states of metals. In the vicinity of a cation vacancy in oxides, valence band top states become usually split off into the bandgap. Therefore, we are inclined to associate the mechanism of transient optical absorption in the borate crystals with photoinduced hole transfer between the $2 p$ states of neighboring anions surrounding a lithium vacancy. Considered in the context of the theory of small-radius polarons, this process is treated as inter-polaron absorption. The profile of the optical absorption spectrum is largely mediated in these conditions by the distribution of the density of valence band states. At $T=290 \mathrm{~K}$, a hole undergoes thermally activated and, possibly, partially tunneling migration among the anions surrounding the vacancy. The energy threshold of optical absorption (1-2.5 eV in figure 1) is actually the energy of an optical transition between the local level of the hole center and the valence band top, i.e., the optical activation energy of the $\mathrm{O}^{-}$hole center. Optical absorption of this type was observed by us earlier in the LTB [3], LBO [4] and LGBO [5] lithium borate crystals.

The concentration of the $\mathrm{O}^{-}$centers responsible for the TOA can decrease not only through the ionic process of diffusion-limited annihilation of lithium vacancies with $\mathrm{Li}^{0}$ interstitial atoms, but in tunneling 
charge exchange of these defects involving tunneling transfer of an electron from the interstitial $\mathrm{Li}^{0}$ atom to the $\mathrm{O}^{-}$center as well. It is this transfer that accounts for the main 'tunneling component' in the TOA decay kinetics. This appears to provide reasonable grounds for the use of the theory of tunneling charge exchange among randomly distributed defects and for fitting Eq. (2) to the TOA kinetics in describing the above component. The probability of tunneling electron transfer between the tunneling recombination partners depends on the pre-factor $v_{0}$ and distance $r$ between the partners. The lithium cation mobility affects the distribution of defect pairs in defect separations $r$ and gives rise to an increase in the 'close pair' concentration. This accounts for the observed temperature dependence of the 'tunneling' component of the TOA decay kinetics.

\section{Conclusion}

Thus, we have carried out a study of the processes of creation and evolution of the short-living defects in the cation sublattice of crystals of the lithium borates LBO, LTB and LGBO. The experimental results were mainly obtained by the use of luminescent and optical spectroscopy with time resolution under excitation with an electronic beam. The analysis carried out in the work allowed us to elaborate a view of the creation and relaxation mechanism of short-living Frenkel's pair of defects in the cation sublattice of the lithium borate crystals.

1. The presence of a sublattice of weakly bonded lithium cations is a common feature, which pools the considered crystals. An effective creation of the Frenkel's pairs "lithium vacancy - interstitial cation" can occur at a comparatively moderate radiation action (a nanosecond-pulse electron beam with an energy of $200-250 \mathrm{keV}$ ). A capture of the charge carriers on these defects leads to a creation of both the trappedhole center $\mathrm{O}^{-}$and the deep trapped-electron center $\mathrm{Li}^{0}$. For crystals with a sublattice of mobile cations this mechanism of creation of defects under the action of ionizing radiation shows up as rather universal.

2. Electronic transitions from the valence band states onto a local level of the trapped-hole center $\mathrm{O}^{-}$ cause the observed short-living optical absorption in these crystals. Taking into account the data on the electronic structure of a valence band, this process can be interpreted as the photoinduced transitions of a hole between the $2 p$-orbitals of the neighbor oxygen ions, surrounding the lithium vacancy.

3. The tunnel transitions of an electron between the lattice defects in the form of the trapped-electron center $\mathrm{Li}^{0}$ and the trapped-hole center $\mathrm{O}^{-}$control the TOA decay kinetics in the decay-time range from nanoseconds to several milliseconds.

4. A thermally stimulated migration of the lithium cations, which occurs even at room temperature, determines the temperature dependence of the TOA decay kinetics. In the long decay time-range, the TOA decay kinetics is mainly controlled by a process of a thermally stimulated migration of the lithium cations and the TOA decay kinetics of all the crystals tends asymptotically to the first order hyperbolic law.

\section{Acknowledgments}

The authors thank L.I.Isaenko, A.V.Tolmachev, R.P.Yavetskiy for providing the samples of lithium borates and V.Yu.Yakovlev for his assistance in the time-resolved optical absorption spectroscopy measurements.

\section{References}

[1] Ogorodnikov I N, Pustovarov V A, Kruzhalov A V, Isaenko L I, Kirm M and Zimmerer G 2000 Physics of The Solid State $42464-472$

[2] Ogorodnikov I N, Pustovarov V A, Omelkov S I, Tolmachev A V and Yavetskiy R P 2007 Optics and Spectroscopy 102 60-67

[3] Ogorodnikov I N, Yakovlev V Y, Kruzhalov A V and Isaenko L I 2002 Phys. Solid State 44 1085-1092

[4] Ogorodnikov I N, Yakovlev V Y and Isaenko L I 2003 Phys. Solid State 45 845-853

[5] Ogorodnikov I N, Poryvay N E, Pustovarov V A, Tolmachev A V, Yavetskiy R P and Yakovlev V Y 2010 Radiat. Measurements 45 336-339 
[6] Ogorodnikov I N, Kudyakov S V, Kuznetsov A Y, Ivanov V Y, Kruzhalov A V, Maslov V A and Ol'khovaya L A 1993 Technical Physics Letters 19 431-432

[7] Porotnikov A V, Ogorodnikov I N, Kudyakov S V, Kruzhalov A V and Votyakov S L 1997 Physics of the Solid State 39 $1224-1227$

[8] Ogorodnikov I N, Isaenko L I, Kruzhalov A V and Porotnikov A V 2001 Radiat. Measurements 33 577-581

[9] Shekhovtsov A N, Tolmachev A V, Dubovik M F, Dolzhenkova E F, Korshikova T I, Grinyov B V, Baumer V N and Zelenskaya O V 2002 J. Cryst. Growth 242 167-171

[10] Yakovlev V Y 1992 Sov. Phys. Solid St. 34 651-654

[11] Chen R and Kirsh Y 1981 Analysis of Thermally stimulated processes (Oxford; New York: Pergamon Press)

[12] Parmon V N, Khairutdinov R F and Zamaraev K I 1974 Sov. Phys. Solid St. 16 1672-1675

[13] Hopfield J J 1974 Proc. Nat. Acad. Sci., USA 71 3640-3644

[14] Malovichko G. I, Grachev V G and Matkovskii A O 1991 Sov. Phys. Solid St. 33 1107-1111

[15] Matkovskii A O, Sugak D Y, Burak Y V, Malovichko G I and Grachev V G 1994 Radiat. Eff. Defect. Solid. 132 $371-376$ 